\title{
Robot-Assisted Smart Firefighting and Interdisciplinary Perspectives
}

\author{
Pengcheng Liu ${ }^{1}$ (PhD student), Student Member, IEEE, Hongnian $\mathrm{Yu}^{1}$, Shuang Cang ${ }^{2}$, and Luige Vladareanu ${ }^{3}$ \\ ${ }^{1}$ Faculty of Science and Technology, Bournemouth University, Poole BH125BB, UK \\ ${ }^{2}$ Faculty of Management, Bournemouth University, Poole BH125BB, UK \\ ${ }^{3}$ Dept. Robotics and Mechatronics, Institute of Solid Mechanics of Romanian Academy, Bucharest, Romania
}

\begin{abstract}
Urbanization and changes in modern infrastructure have introduced new challenges to current firefighting practices. The current manual operations and training including fire investigation, hazardous chemicals detection, fire and rescue are insufficient to protect the firefighter's safety and life. The firefighting and rescue functions of the existing equipment and apparatus and their dexterity are limited, particularly in the harsh firefighting environments. It is well-established that data and informatics are key factors for efficient and smart firefighting operation. This paper provides a review on the robot-assisted firefighting systems with interdisciplinary perspectives to identify the needs, requirements, challenges as well as future trends to facilitate smart and efficient operations. The needs and challenges of robot-assisted firefighting systems are firstly investigated and identified. Subsequently, prevailing firefighting robotic platforms in literature as well as in practices are elaborately scrutinized and discussed, followed by investigation of localization and navigation support methods. Finally, conclusions and future trends outlook are provided.
\end{abstract}

Keywords-Firefighting Robotic Platforms; Localization and Navigation; Sensors; Autonomy; Resilience.

\section{INTRODUCTION}

Over the years, throughout the world, fire losses remain high and firefighting is strenuous and dangerous. Training and research programs have been developed to confront the challenges in firefighting, whilst there are still significant losses from fires each year. The entire direct and indirect cost of fire losses as a percentage of Gross Domestic Product in the world is estimated to up to $1 \%$ annually [1]. The US fire departments responded to over 1,240,000 fires in 2013, which resulted in approximately 3,420 civilian fatalities, 15,925 injuries and property losses of about $\$ 12.4$ billion dollars [2]. Exposure to harsh conditions on fire ground, such as smoke inhalation, fire burns, overexertion/stress, or even being trapped, is considered to be the main attributions to more than $60 \%$ of the firefighter deaths and over $20 \%$ firefighter injuries [3], [4].

Robotics are playing important roles intelligently and technologically that assist emergency responses in harsh and hazardous firefighting environments whilst prevent operational personnel from entering inaccessible or unsafe regions. A considerable body of unmanned remotely driven response robots has proven to lower certain risks for the emergency team. They assist the responders and the experts in new and innovative ways. This paper reviews the state-of-art in robot-assisted firefighting to identify the challenges, requirements and trends in this field and provides interdisciplinary perspectives to facilitate smart firefighting in the future.

The rest of the paper is organized as follows. Section II provides the needs and challenges of robot-assisted firefighting systems. The discussion on latest development of firefighting robotic platforms is presented in Section III. Section IV investigates the state-of-art in location and navigation support methods. Finally, conclusions and future trends are given in Section V.

\section{NEEDS, REQUIREMENTS AND CHALLENGES OF ROBOT- ASSISTED FIREFIGHTING SYSTEMS}

During the last two decades, there is a growing conception in both scientific and technological domains that being "smart" means to significantly enhance the autonomy of the system, in a manner that troublesome human errors can be sufficiently avoided. As indicated in [5], the concept of "smart" should contain the system autonomy and, more importantly, system resiliency to many possible internal disturbances as well as external structured and unstructured dynamics. In this regard, smart can be featured as physical and cognitive integration and interactions of humans, machines as well as organizations to boost the system performance and manipulate the system resilience.

Exposure to the hazardous and chaotic fire environment, rather than to the fire itself, is the most significant cause of injury and death in fires. The reachability of precise information in real-time on the conditions directly at the centre of the fire ground is a crucial factor in the guidance of rescue actions together with feasible counter-plans. Unfortunately the firefighting environments are normally hard to reach and restricted in accessibility by obstacles, tumbledown architectures and visibility by smoke, dangerous 
gasses or dust. Therefore, the fire scene is an information-poor environment due to lack of information on location of fire, firefighters and victims, and the search and rescue opportunities are previously unimaginable due to lack of situational conditions and real-time information for targeted decision making. It is found that a restricted visual field and obscured cameras augments the distress of firefighters working under pressure. The exposure time of individuals and unobstructedness of firefighting and rescue paths are of paramount importance for the operational efficiency of firefighting and rescue particularly during and after the incidences. The term unobstructedness refers to the guarantee of adaptability to the physical environments change, for instance, ceilings, floors, or walls collapse, furniture relocates. The prevailing high-tech localization and navigation systems are generally not adaptable to these dynamic changes, wherein thick smoke, high temperature, gusts of air, noise, obstacles and falling debris hinder the propagation of the ultrasound, radio, and laser signals conventionally utilized for localization and perception [6]. In smoke laden circumstances with restricted visibility, the exposure time is extremely relying on the dynamic knowledge of the growing fire and the three dimensional movement of smoke within such environments. Human behaviours (e.g. initial response, movement redirections as well as walking speed [7], [8]) make firefighting operation difficult to conduct [9], [10]. A large amount of research has been devoted regarding human behaviour in fires and the simulation of the movement of individuals in such hazardous environment. To date, the prediction and sensing of visibility are typically reliant upon empirical and static data from preinstalled and infrastructurebased location systems for various targets and do not take into account the hybrid dynamic concentrations, wherein those systems typically fail in environmental conditions changes (e.g. temperature rises, furniture moves, floors collapse), and power failure.

The entry time of firefighters and escaping time of individuals are largely depending on the unobstructedness of firefighting and rescue paths. Currently, robots do not sufficiently enhance human confidence. Necessary capabilities to such robots are the perceived visibility of the surroundings, heavy physical tasks (HPT) such as obstacle avoidance, forcible entry, sweeping and loading to guarantee the paths unobstructedness. Ideally, these capabilities should be available as a function of the dynamic fire environment. To date, the functionalities of the firefighting robots are restricted into information collection, flame detection, remotely fire extinguishing, etc., and conventionally no heavy physical tasks are assigned. Despite considerable advancements in the development of sensor technologies and robotics for firefighting there has been little interaction between robots and human perceptions of visibility as recorded in field trials and the equivalent numerical infrastructure simulations of visibility in a dynamic firefighting scene.

\section{FIREFIGHTING ROBOTIC PLATFORMS}

Fire, smoke, darkness, water, power outages and noise may hinder a firefighting, searching and rescue system from working, and the personal protective equipment (PPE), gloves as well as facemasks prevent standard mobile computers from working [6]. Conceptually, a smart firefighting and rescue system should contain risky intervention, information gathering, storage, exchange, analysis, and integration from a variety of sensor networks and dynamic databases for environmental surveillance, decision recommendation and support. Challenges and difficulties are associated with each of the aforementioned domains.

The SnakeFighter Anna Konda [11], [12] shown in Fig. 1 is able to push against external obstacles apart from a flat ground and capable of obstacle-aided locomotion and extinguishing fire using a nozzle mounted at the front of the robot, with hydraulic medium in the joint actuation. A combined utilization of water is realized for hydraulic joint actuation, fire extinguishment and robot cooling under high temperature.

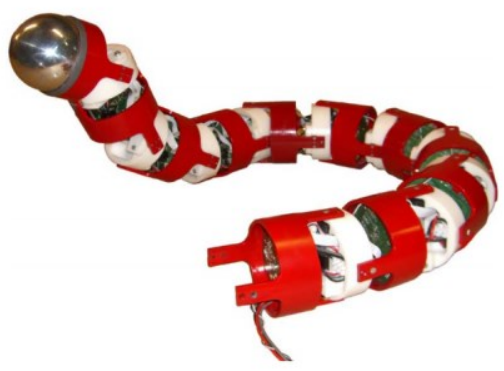

Fig. 1. The SnakeFighter Anna Konda [11], [12].

LUF60 [13] shown in Fig. 2 is a popular firefighting robot equipping with an air blower and a water beam fog. The monitor nozzle has a flow rate up to 800 GPM and it is capable of blowing the water beam up to $80 \mathrm{~m}$. For the sake of enhancing the mobility in the harsh condition of high temperature, rubber track system is equipped with heat resistance rate up to 400 degrees Fahrenheit. The rubber track system also enables the capability of descending and ascending the stairs.

A humanoid firefighting robot, SAFFiR [14] shown in Fig. 3 , utilizes a bio-inspired geometry with parallel actuated biped using linear actuators, it is capable of omnidirectional walking, balancing in sea state conditions, traversing obstacles and manipulating fire suppressors.

Parosha Cheatah GOSAFER [15] is designed for rough terrain and capable of operating in several environmental conditions using a 10 road wheels for high mobility. A mixture of water and cutting agent is equipped and being ejected via a nozzle on a lance at high pressure to cut through most of the known construction materials very quickly. A powerful firefighting robot TAF 20 shown in Fig. 4 [16] can sweep away the obstacles via bulldozer blades and clear 
smoke from burning buildings with a high-powered fan. It is also able to spray water mist or foam from $60 \mathrm{~m}$ and blast water for $90 \mathrm{~m}$. Remote control and operation can be facilitated up to $500 \mathrm{~m}$ away to send the robot into environments hazardous to firefighters.

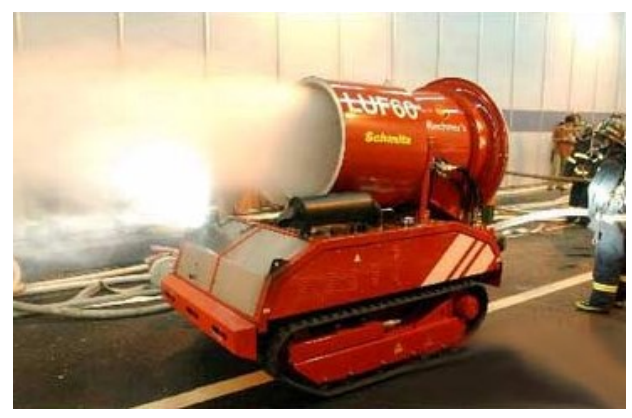

Fig. 2. LUF60 [13].

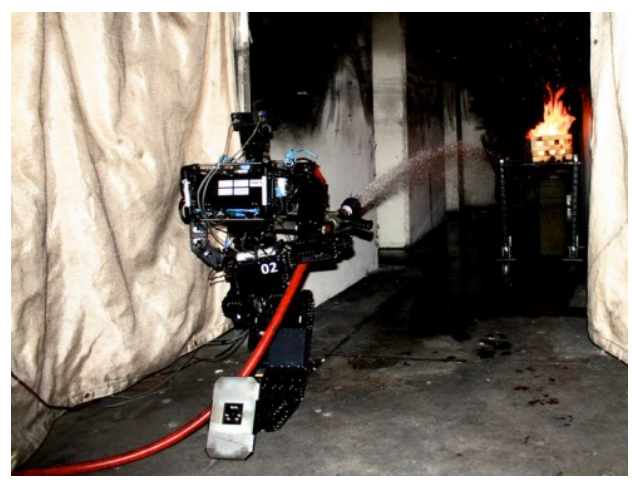

Fig. 3. SAFFiR [14].

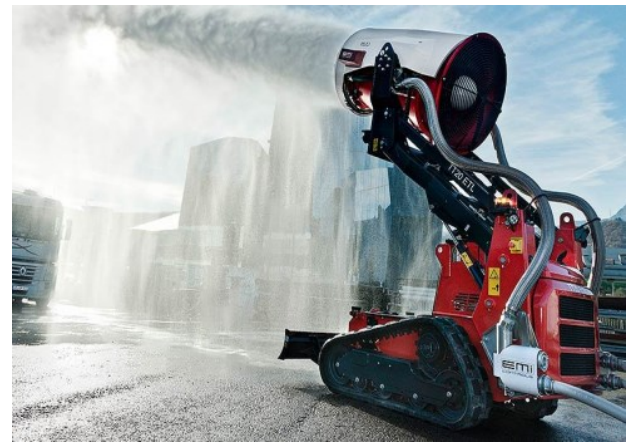

Fig. 4. TAF 20 [16].

Thermite 3.0 [17] is a small firefighting robot is capable of fitting into restricted space. It has integrated multiple HD analogy video cameras and optional Infrared (IR) FLIR. As an electrically powered Unmanned Ground Vehicle (UGV), FIREMOTE [18] can be remotely operated through a control panel and tracked outside the dangerous area. The control panel has a daylight viewable monitor that a software dashboard can be displayed with the robot's parameters as well as video feedback for navigation. FIREMOTE is equipped with a monitor, colour navigation camera, local cooling system and variable pattern nozzle. ArchiBot-M [19] has an independent suspension system. It is capable of ascending and descending stairs and working under high temperatures, since it is waterproof and equipped with a cooling system. More interestingly, the Sweden-made firefighting robot Brokk50 [20] is capable of forcible entry, rescue, excavation, notching, carrying payload within unsafe or extreme environment.

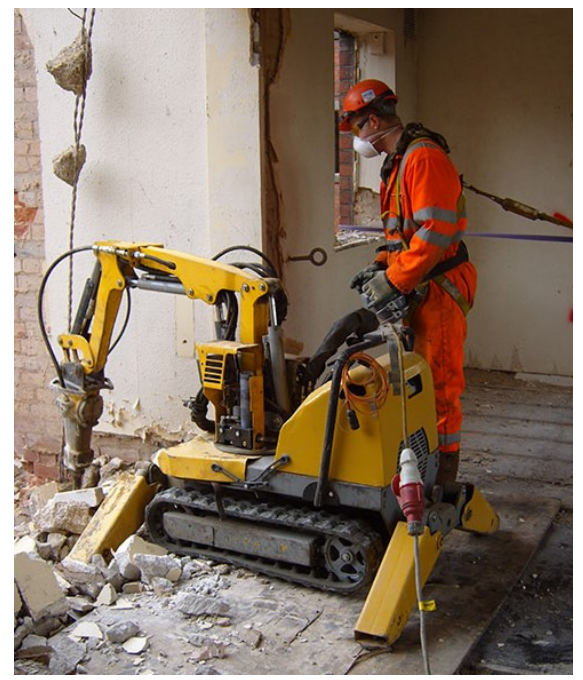

Fig. 5. Brokk50 [20].

The comparison of the prevailing robot-assisted firefighting platforms with key features is presented in Table I. The emerging and prosperous developments of robotics, ICT technologies as well as data informatics have provided a variety of effective and promising solutions to many practical problems in emergency responses. However, open challenges and difficulties alongside huge, complex and tough tasks associated with emergencies in firefighting are still far from being fully addressed. The requirements and challenges are identifies as follows:

- Traversability and dexterity: Higher degree of traversability and dexterity of firefighting robotic platforms are required for the ease of accessing and operating in areas inaccessible and hostile to humans.

- Heat/Radiation resistance: The firefighting operations require the robots to be resistant to heat and radiation and to have impact resistance mechanisms. Besides, the on-board equipment and apparatus such as sensors, machine tools and other on-board components must be heat resistant as well or cooling systems is necessary to provide protection such as water-based cooling system.

- $\quad$ Supervised and semi-supervised autonomy with effective human-robot (H2R) teamwork: The robots are required to be controllable via some intuitive $H 2 R$ interfaces. The robots should be equipped with sensory capabilities (e.g., temperature/gas/pressure/noise sensor, sonar, radar, and camera) and machine tools (e.g., 
gripper, welding tool, and fire hose nozzle) in compatible with given tasks. Besides, the robots should be able to monitor the fire ground situation and meanwhile, report the collected information to firefighters and command centre.

- Portability: In most circumstances, firefighters are sent to the fire ground as soon as a fire or other emergency is reported. Thus, the firefighting robot needs to be lightweight and convenient to carry to rapidly cope with the fire emergency. Besides, it is also important that the deployed robots can be safely withdrawn by firefighters.

TABLE I. COMPARISON OF FIREFIGHTING ROBOTIC PLATFORMS WITH KEY FEATURES

\begin{tabular}{|c|c|c|c|c|c|c|c|c|}
\hline Robot & Type & $\begin{array}{l}\text { Operating } \\
\text { Region }\end{array}$ & Capabilities & Operating Mode & Perception & Portability & HPT & $\begin{array}{l}\text { Country } \\
\text { of Origin }\end{array}$ \\
\hline $\begin{array}{l}\text { Anna } \\
\text { Konda }{ }^{[11][12]}\end{array}$ & $\begin{array}{l}\text { Snake-like } \\
\text { robot }\end{array}$ & $\begin{array}{l}\text { Indoor/ } \\
\text { Outdoor }\end{array}$ & $\begin{array}{l}\text { Fire extinguishment/ } \\
\text { Visual perception }\end{array}$ & $\begin{array}{l}\text { Obstacle-aided/ } \\
\text { Autonomous }\end{array}$ & $\begin{array}{l}\text { Thermometer/ } \\
\text { Visual camera }\end{array}$ & Medium & Poor & Norway \\
\hline LUF60 $^{[13]}$ & UGV & Outdoor & $\begin{array}{l}\text { Fire extinguishment/ } \\
\text { Smoke dispelling/ } \\
\text { Stair climbing }\end{array}$ & $\begin{array}{l}\text { Remote control/ } \\
\text { Rubber track system }\end{array}$ & Visual camera & Poor & Poor & Germany \\
\hline SARRIR $^{[14]}$ & $\begin{array}{l}\text { Humanoid } \\
\text { robot }\end{array}$ & Indoor & $\begin{array}{l}\text { Omnidirectional } \\
\text { walking/ Manipulating } \\
\text { fire suppressors }\end{array}$ & $\begin{array}{l}\text { Parallel actuated/ } \\
\text { Autonomous }\end{array}$ & $\begin{array}{l}\text { Thermal IR stereo/ } \\
\text { Visual camera }\end{array}$ & Poor & Medium & $\begin{array}{l}\text { United } \\
\text { States }\end{array}$ \\
\hline $\begin{array}{l}\text { Parosha } \\
\text { Cheatah } \\
\text { GOSAFER }^{[15]}\end{array}$ & UGV & Outdoor & $\begin{array}{l}\text { Fire extinguishment/ } \\
\text { Cutting Extinguisher/ }\end{array}$ & $\begin{array}{l}\text { Remote control/ } \\
\text { Rubber track system }\end{array}$ & $\begin{array}{l}\text { Thermal image camera/ } \\
\text { Laser range finder/ } \\
\text { Acoustic detection }\end{array}$ & Poor & Medium & Sweden \\
\hline TAF $20^{[16]}$ & UGV & Outdoor & $\begin{array}{l}\text { Fire extinguishment/ } \\
\text { Smoke dispelling/ } \\
\text { Obstacles sweeping }\end{array}$ & $\begin{array}{l}\text { Remote control/ } \\
\text { Rubber track system }\end{array}$ & N.A. & Poor & High & Germany \\
\hline $\begin{array}{l}\text { Thermite } 3.0 \\
\text { [17] }\end{array}$ & UGV & $\begin{array}{l}\text { Indoor/ } \\
\text { Outdoor }\end{array}$ & Fire extinguishment/ & $\begin{array}{l}\text { Remote control/ } \\
\text { Rubber track system }\end{array}$ & $\begin{array}{l}\text { HD analog video } \\
\text { cameras/ } \\
\text { Optional Infrared FLIR }\end{array}$ & Medium & Poor & $\begin{array}{l}\text { United } \\
\text { States }\end{array}$ \\
\hline $\begin{array}{l}\text { FIREMOTE } \\
{[18]}\end{array}$ & UGV & Outdoor & $\begin{array}{l}\text { Fire extinguishment/ } \\
\text { Visual perception }\end{array}$ & $\begin{array}{l}\text { Remote control/ } \\
\text { Rubber track system }\end{array}$ & Visual camera & Poor & Poor & $\begin{array}{l}\text { United } \\
\text { Kingdom }\end{array}$ \\
\hline Archibot $^{[19]}$ & UGV & Indoor & $\begin{array}{l}\text { Fire extinguishment/ } \\
\text { Stair climbing }\end{array}$ & Remote control & Visual camera & Medium & Poor & $\begin{array}{l}\text { South } \\
\text { Korea }\end{array}$ \\
\hline Brokk $^{[20]}$ & $\begin{array}{l}\text { Excavator- } \\
\text { like robot }\end{array}$ & $\begin{array}{l}\text { Indoor/ } \\
\text { Outdoor }\end{array}$ & $\begin{array}{l}\text { Demolition/ } \\
\text { Stair climbing }\end{array}$ & $\begin{array}{l}\text { Remote control/ } \\
\text { Hybrid locomotion }\end{array}$ & N.A. & Medium & High & Sweden \\
\hline
\end{tabular}

- $3 D$ perception: In smoke laden circumstances with restricted visibility, the robots need to be capable of acquiring the dynamic knowledge of the growing fire and the three dimensional movement of smoke within such environments.

- Heavy physical tasks: The robots should be designed to perform heavy physical tasks such as heavy payload, obstacle sweeping and force entry when and where needed particularly in search and rescue.

- Flame detection and fire extinguishing: A firefighting robotic platform should have the ability of assisting firefighters in fire flame detection and extinguishing with launched or on-board extinguishing medium.

- Situation awareness and intuitive control: the firefighting robotic systems should have the capabilities to communicate with the firefighters and to facilitate machine-to-machine (M2M) communications.

- Dexterous manipulation and maneuverability: A robotic system needs to have the capability of dexterous manipulation and high degree of maneuverability to cope with the rough terrain in emergency response, such as omnidirectional driving, adaptation to dynamic uncertainties, climbing over obstacles, ascending and descending stairs, etc.

\section{LOCALIZATION AND NAVIGATION SUPPORT}

The emergency operational conditions for firefighters are extremely more demanding than non-emergency conditions. The environmental circumstances of darkness, thick smoke, high temperature, fire, power outage, water and noise, and the individual circumstance of firefighters with heavy PPE, gloves and facemasks all hindered the location system and standard portable computers from working. The timely and safe reachability of the emergency response team before the situations become too hazardous is of vital importance, and the locations of team members must be tracked by the incident commanders (ICs) in real-time. The needs and requirements for localization and navigation systems suggested by $\mathrm{NIOSH}$ [21] are listed as follows:

- Ensure that the ICs continuously evaluates the risk versus gain when determining whether the fire suppression operation will be offensive or defensive.

- Ensure that the ventilation to release heat and smoke is closely coordinated with interior fire suppression operations. 
- Ensure that firefighters wear a full array of turnout clothing and PPE suitable for the assigned task while participating in firefighting activities.

- Consider using thermal imaging cameras (TICs) during the initial size-up and search phases;.

- Develop and refine durable, easy-to-use radio systems to enhance verbal and radio communication in conjunction with properly worn self-contained breathing apparatus (SCBA).

- Conduct research into refining existing and developing new technology to track the movement of fire fighters inside structures.

The emergency response teams have developed practical localization and navigation methods used in restricted visibility. The current low-tech practices contain following a hose or dedicated ropes (lifelines) to remain physically linkage with other team members. The knots along the lifeline help firefighters determine the direction and distance to the exit and can be used as reference points when sending position information to ICs [22]. Besides, Personal Alert Safety System (PASS) device is prevailingly used attaching to firefighters' breathing apparatus when they enter hazardous areas.

\section{A. Sensors}

Sensors are capable of transforming physical environmental characteristics in fire emergencies into raw data, and subsequently enabling the process of converting raw data into information which are actionable. The sensors can be categorized into several groups as summarized and discussed in the following.

1) UV detectors [23] and UV light emitting diodes [24]: They are mostly used for early stage fire detection due to their sensitivity to UV, and they are able to detect long ranges UV radiation emitted. The demerit is that it cannot fix the position since the sensor view field is large.

2) Smoke detector: It is a prevailingly used and, to some extent most informative, sensor technology associated with the fire safety in buildings [25].

3) Light detection and ranging (LIDAR): Fire experiments show that LIDAR and visual cameras are typically adopted for robot navigation, whilst signals from LIDAR are attenuated within a visibility of $4 \mathrm{~m}$ or even weaker with no signal within a smoke visibility of $1 \mathrm{~m}$ [26].

4) Visual camera: Visual cameras are normally used to accumulate motion features for smoke and fire detection and video surveillance. However, it is not suitable for fire smoke environments [27].

5) Frequency modulated-continuous wave (FMCW) radar: For the sake of locating objects and humans through smoke, FMCW is typically integrated with a gyroscope and accelerometer and has been widely used in smoke laden environments for 3D imaging and perception [28]. Nevertheless, FMCW radar is only able to provide 1D scanning and it requires more than $20 \mathrm{~s}$ to scan the environmental space mechanically to construct the 3D map. Therefore, real-time perception is of vital importance for robotic systems to conduct object detection and localization for efficient navigation support. The real-time perception system with fused FMCW radar and stereo IR [29] shown in Fig.6 provides a feasible option.

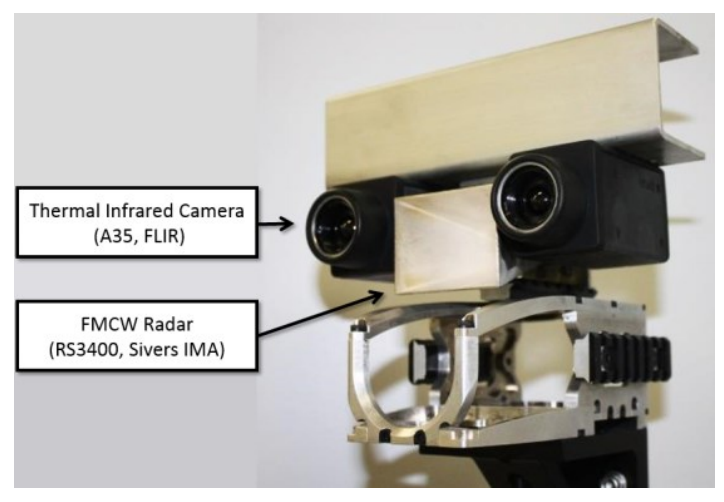

Fig. 6. Real-time perception system with fused FMCW radar and stereo IR developed by Virginia Tech [29].

6) Sonar/Ultrasonic sensor: Accurate measurement of the distance is unavailable through fire smoke due to the fact that the sound speed changes with the gas temperature of the smoke varied [30].

7) RGB-depth sensor (Kinect ${ }^{T M}$ ): The attenuation of the speckle pattern through smoke and the receiving radiation on the sensor from the fire interfering with the return speckle pattern result in the unavailability of 3-D imaging of the scene [26].

8) Thermal infrared cameras: It becomes prevailing integral equipment for the fire service using in structure fires and other emergencies. It performs well both in clear and smoke laden environments when operating with long wavelength infrared range $(7-14 \mu \mathrm{m})$ and the radar at a wavelength about $11.5 \mathrm{~mm}$ [26]. Employing these technologies, images can be obtained via features of highlight high temperature and obscurant with high concentrations [31]. For instance, using satellites to monitor the forest fires [32], computing the rate of spread of linear flame fronts [33], detection of tunnel fires [34], ground installed cameras [35]. The applications above percept the targeting environments with 2D views, however to locate targeted fires, 3D information are crucial and necessary. It turns out that stereo vision system could be one option, whilst it has several demerits to be overcome [31]: a) its accuracy decreases with the distance increases; b) it takes some time to generate images with full resolution; c) in the generated disparity map, both existing and non-existent objects (i.e., ghost objects) are existed owing to the stereo mismatches.

\section{B. Localization and navigation support systems}

The localization and navigation support systems for firefighting have been built alongside the advancements of the 
technologies. The prevailing methods among them are listed and discussed in the following.

1) Infrastructure-based method (Preinstalled location systems): A great number of indoor localization systems measure the distance and angle information to locate the coordinates of a target. The target is typically a tag transmitting signal of ultrasound, radio or IR which are detectable. The sensor collects the distance or direction information of the signal, and the position of the tag is estimated by a central computer through trilateration or triangulation. The estimated positions are normally sufficiently accurate for reconstruct the floor plan.

Merits: The performance of update rate, reliability can be very good.

Demerits: This method is not robust to environmental changes such as temperature rises, floors collapse or power lost, etc.

2) Wireless sensor networks: The optimization of deployments of sensors in wireless sensor networks, as application-specific systems, has been advanced by various researchers. The measurements of range or connectivity are shared between sensors and their locations can be recognized via calculations from each sensor without the endeavour from the central device.

Merits: a) No reliance on central devices; b) Only the positions of the anchor nodes need to be calibrated and all the other nodes can just be scattered or dropped without calibrations; c) No infrastructure is needed ascribe to the distributed computation and wireless communication; d) Failure may occur to individual nodes instead of compromising the entire system.

Demerits: If sensors are subject to dynamic movements from their initial positions, the algorithms with densely distribution of nodes might provide incorrect estimates of the position.

3) Ad-Hoc Relative Positioning: This method is on the basis of the claim that the angular measurements between devices can be achieved through physically rotating the receiver or transmitter, or using an array of receivers or transmitters [36].

Merits: Its principle is simple using the measurements without complicated processing.

Demerits: The reliability of measurements needs to be guaranteed. In some circumstances, single measurements might be unreliable because the signals are reflected off surfaces or obstructed by obstacles.

4) Proximity Sensing: RFID tages can be detected and identified by RFID readers within a certain range. The positions of readers can be estimated when the tag's position is known. The accuracy of estimation increases when a shorter detection range is given whilse more tags are required.
Merits: This method is cost-effective with small size, and a power source is not required, and it is convenient to implement such as embedded under the carpet tiles.

Demerits: Calibrations are required to identify position of each tag.

To integrate sensor data with analytics tools in software within and across architectural levels, the following requirements [37] need to be satisfied:

- Standardized networking protocols to cover the wireless communications;

- Standardized syntax and semantics to cover the conceptual content.

In the circumstance of firefighting, the expert understanding of multidisciplinary knowledge is needed, including engineering in fire protection, physics, fire science, and information science. So far, the explorations of information modelling in those disciplines have been virtually non-existent. The effectiveness of communication and therefore H2R and $\mathrm{M} 2 \mathrm{M}$ on the fire ground are often problematic due to the fact that the quality and quantity of data and information are highly variable and unreliable.

\section{CONCLUSIONS AND FUTURE TRENDS}

Firefighting is an evolving research area, benefitting from rapid advancements of technologies and driven by our ongoing pursuit for robot-assisted risky-intervention, localization and navigation, early rescue as well as environmental surveillance. This paper gave an overview of the state-of-art in robotassisted smart firefighting systems, the localization and navigation support methods and discussed the potential applications for the ease of realizing smartness in emergency responses towards firefighting.

Conventional firefighting robotic platforms and traditional methods for localization and navigation support have limited capabilities and performance for firefighting operation. To facilitate sufficient autonomy as well as resiliency during firefighting practices, humans' critical role needs to be realized through the advancements of $\mathrm{H} 2 \mathrm{R}$ and $\mathrm{M} 2 \mathrm{M}$ interactions. In this regard, the emerging Cyber Physical Systems (CPSs) technology becomes crucial enabling factors, which feature a tight combination of, and coordination between, the computational and physical elements of the system and integration of computer and information-centric physical and engineered systems. The introduction and integration of appropriate and promising technologies and systems will therefore facilitate the utilization and fusion of a wide range of real-time information and data not only during the fire emergency incidents but also the pre-incidents and postincidents. These data and information can be potentially provide valuable input for decision support systems and therefore enhance the efficiency of fire protection and firefighting operation. 


\section{ACKNOWLEDGMENT}

The part of this work is supported by RABOT, http://rabot.fusion-edu.eu/ (PIRSES-GA-2012-318902).

\section{REFERENCES}

[1] B. Woodrow, "A Cautionary Note on Comparative World Fire Statistics, and Specifically the Case of the Russian Wildfires of 2010," World Fire Stat., vol. 27, 2011.

[2] "World fire statistics | CTIF - Comité technique international de prévention et d'extinction du Feu." [Online]. Available: http://www.ctif.org/ctif/world-fire-statistics. [Accessed: 21-Apr-2016].

[3] M. J. Karter, Fire loss in the United States during 2012. NFPA, 2013.

[4] R. F. Fahy, P. R. LeBlanc, and J. L. Molis, Firefighter Fatalities in the United States-2011, vol. 1. NFPA, 2012.

[5] A. Jones, E. Subrahmanian, A. Hamins, and C. Grant, "Humans' Critical Role in Smart Systems: A Smart Firefighting Example," Internet Comput. IEEE, vol. 19, no. 3, pp. 28-31, 2015.

[6] C. Fischer and H. Gellersen, "Location and navigation support for emergency responders: A survey," IEEE Pervasive Comput., vol. 9, no. 1, pp. 38-47, 2010.

[7] J. Shi, A. Ren, and C. Chen, "Agent-based evacuation model of large public buildings under fire conditions," Autom. Constr., vol. 18, no. 3, pp. 338-347, 2009

[8] K. Fridolf, E. Ronchi, D. Nilsson, and H. akan Frantzich, "Movement speed and exit choice in smoke-filled rail tunnels," Fire Saf. J., vol. 59, pp. 8-21, 2013.

[9] E. Ronchi, S. M. V. Gwynne, D. A. Purser, and P. Colonna, "Representation of the impact of smoke on agent walking speeds in evacuation models," Fire Technol., vol. 49, no. 2, pp. 411-431, 2013.

[10] M. S. Wright, G. K. Cook, and G. M. B. Webber, "The effects of smoke on people's walking speeds using overhead lighting and Wayguidance provision. Human behavior in fire," in Proceedings of the 2nd international symposium, MIT, 2001.

[11] A. A. Transeth, R. I. Leine, C. Glocker, K. Y. Pettersen, and P. LiljebÄck, "Snake Robot Obstacle-Aided Locomotion: Modeling, Simulations, and Experiments," IEEE Trans. Robot., vol. 24, no. 1, pp. 88-104, Feb. 2008.

[12] P. Liljeback, O. Stavdahl, and A. Beitnes, "SnakeFighter - Development of a Water Hydraulic Fire Fighting Snake Robot," in 9th International Conference on Control, Automation, Robotics and Vision, 2006. ICARCV '06, 2006, pp. 1-6.

[13] "Löschunterstützung - LUF Fire-Fighter." [Online]. Available: http://www.luf60.at/loeschunterstuetzung/. [Accessed: 07-Apr-2016].

[14] "Shipboard Autonomous Firefighting Robot (SAFFiR) - Office of Naval Research." [Online]. Available: http://www.onr.navy.mil/en/MediaCenter/Fact-Sheets/Shipboard-Robot-Saffir.aspx. [Accessed: 16-Mar2016].

[15] "Fire Fighting UGV | Parosha Cheatah GOSAFER.".

[16] "Trucked vehicle TAF20 | Emission control." [Online]. Available: http://www.emicontrols.com/english/Fire\%20fighting/Solutions/Trucked \%20vehicle\%20TAF20.html. [Accessed: 16-Mar-2016].

[17] "Fire Fighting Robot: THERMITE 3.0," Fire Fighting Robot: THERMITE 3.0. [Online]. Available: http://www.firefightrobot.com/. [Accessed: 16-Mar-2016].

[18] "Firemote." [Online]. Available: http://www.rylandresearch.co.uk/unmanned-vehicles/firemote. [Accessed: 16-Mar-2016].

[19] “::: Welcome to DRB Fatec :::" [Online]. Available: http://www.drbfatec.com/frd_center/fighting_m.htm. [Accessed: 16Mar-2016].

[20] "Brokk 50 - remote controlled demolition robot." [Online]. Available: http://www.brokk.com/us/50/. [Accessed: 16-Mar-2016].

[21] "CDC - Fire Fighter Fatality Investigation and Prevention Program: Main Page - NIOSH Workplace Safety and Health Topic.” [Online]. Available: http://www.cdc.gov/niosh/fire/. [Accessed: 10-Apr-2016].
[22] T. E. Sendelbach, "Search Line Survival Training," Chief Train. Savannah Fire Emerg. Serv., 2002.

[23] M. K. Rangan, S. M. Rakesh, G. S. P. Sandeep, and C. S. Suttur, “A computer vision based approach for detection of fire and direction control for enhanced operation of fire fighting robot," in Control, Automation, Robotics and Embedded Systems (CARE), 2013 International Conference on, 2013, pp. 1-6.

[24] G. Ariyawansa, M. B. M. Rinzan, M. Alevli, M. Strassburg, N. Dietz, A. G. U. Perera, S. G. Matsik, A. Asghar, I. T. Ferguson, H. Luo, and others, "GaN/ AlGaN ultraviolet/infrared dual-band detector," Appl. Phys. Lett., vol. 89, no. 9, p. 091113, 2006.

[25] A. Cowlard, W. Jahn, C. Abecassis-Empis, G. Rein, and J. L. Torero, "Sensor Assisted Fire Fighting," Fire Technol., vol. 46, no. 3, pp. 719741, Dec. 2008

[26] J. W. Starr and B. Y. Lattimer, "Evaluation of navigation sensors in fire smoke environments," Fire Technol., vol. 50, no. 6, pp. 1459-1481, 2014.

[27] F. Yuan, "An integrated fire detection and suppression system based on widely available video surveillance," Mach. Vis. Appl., vol. 21, no. 6, pp. 941-948, 2010.

[28] J.-H. Kim, J. W. Starr, and B. Y. Lattimer, "Firefighting Robot Stereo Infrared Vision and Radar Sensor Fusion for Imaging through Smoke," Fire Technol., vol. 51, no. 4, pp. 823-845, 2015.

[29] Z. Yang, J. Xijing, J. Teng, Z. Zhu, L. Hao, and W. Jianqi, "Detecting and identifying two stationary-human-targets: a technique based on bioradar," in Pervasive Computing Signal Processing and Applications (PCSPA), 2010 First International Conference on, 2010, pp. 981-985.

[30] A. Purohit, Z. Sun, F. Mokaya, and P. Zhang, "SensorFly: Controlledmobile sensing platform for indoor emergency response applications," in Information Processing in Sensor Networks (IPSN), 2011 10th International Conference on, 2011, pp. 223-234.

[31] J. G. McNeil and B. Y. Lattimer, "Autonomous Fire Suppression System for Use in High and Low Visibility Environments by Visual Servoing," Fire Technol., pp. 1-26.

[32] R. Lasaponara, V. Cuomo, M. F. Macchiato, and T. Simoniello, "A selfadaptive algorithm based on AVHRR multitemporal data analysis for small active fire detection," Int. J. Remote Sens., vol. 24, no. 8, pp. 1723-1749, 2003.

[33] E. Pastor, A. Àgueda, J. Andrade-Cetto, M. Muñoz, Y. Pérez, and E. Planas, "Computing the rate of spread of linear flame fronts by thermal image processing," Fire Saf. J., vol. 41, no. 8, pp. 569-579, 2006.

[34] D. Wieser and T. Brupbacher, "Smoke detection in tunnels using video images," NIST Spec. Publ. SP, pp. 79-90, 2001.

[35] P. J. Thomas and O. Nixon, "Near-infrared forest fire detection concept," Appl. Opt., vol. 32, no. 27, pp. 5348-5355, 1993.

[36] M. Hazas, C. Kray, H. Gellersen, H. Agbota, G. Kortuem, and A. Krohn, "A relative positioning system for co-located mobile devices," in Proceedings of the 3rd international conference on Mobile systems, applications, and services, 2005, pp. 177-190.

[37] A. Hamins, N. Bryner, A. Jones, G. Koepke, C. Grant, and A. Raghunathan, "Smart Firefighting Workshop Summary Report March 24-25, 2014 Arlington, Virginia," National Institute of Standards and Technology, NIST SP 1174, Aug. 2014. 